\title{
Validation of an Additively Manufactured Resistojet through Experimental and Computational Analysis
}

\author{
F. Romei ${ }^{a *}$ and A. N. Grubišića \\ ${ }^{a}$ University of Southampton, Southampton, SO17 1BJ, UK
}

\begin{abstract}
This paper presents the first proof of concept validation of the STAR thruster prototype. The device contains an innovative multifunctional monolithic heat exchanger, enabled by metal additive manufacturing processes. A 316L stainless steel printed thruster is characterized through a combination of dry heating and wet firing tests. This includes verification testing with argon in both cold and hot firing mode, at a range of electrical power inputs. Thrust measurements range from $9.7 \mathrm{mN} \pm 0.16 \mathrm{mN}$ to 29.8 $\mathrm{mN} \pm 0.16 \mathrm{mN}$, with a maximum measured specific impulse of $80.11 \pm 1.49$ s. Thrust performance is measured using a highprecision balance, and liquid-metal power transfer terminals to eliminate thermal drift. Highly coupled multiphysics computational models provide validation of the electro-thermal and thermo-fluidic characteristics of the prototype, including a prediction of the maximum propellant stagnation temperature and structural temperature, which were $649^{\circ} \mathrm{C}$ and $854^{\circ} \mathrm{C}$.
\end{abstract}

Keywords: resistojet; all-electric spacecraft; selective laser melting; performance testing; multiphysics; high-temperature.

\section{Introduction}

The current state of the art xenon resistojet has shown specific impulse (ISP) with xenon propellant up to $48 \mathrm{~s}$ utilised for small 1. spacecraft below $500 \mathrm{~kg}$ [1-3]. Such performance can result in either propellant mass savings or increased capability with respect to cold gas thrusters [4]. The primary application for a high performance resistojet is for primary propulsion on small satellite platforms [5] with an emerging possibility of utilisation as a secondary propulsion system for all-electric telecommunication satellite platforms, where a complement of thrusters would form a reaction control system (RCS) using xenon as a common propellant in combination with a primary electric propulsion system [6].

A high-temperature resistojet concept, named the Super-High Temperature Additive Resistojet (STAR), under development at the University of Southampton [4] has a target ISP $>80 \mathrm{~s}$, with an overall thruster efficiency of $>60 \%$. The primary technology lies in the multifunctional heat exchanger, which is 3D printed via Selective Laser Melting (SLM). Fig. 1 shows a schematics of the first heat exchanger design reported in [4] (overall length of $8 \mathrm{~mm}$ and maximum diameter of $16.5 \mathrm{~mm}$ ), which consists of a monolithic thin-wall concentric exchanger, which also serves as a resistive heater and regenerative heat recuperator, where the inner cylinder (1) also integrates a converging-diverging nozzle. The gas flows from the outer channel (1) and recirculates around the heat exchanger until it reaches channel (4), which terminates in the nozzle inlet. The four concentric cylinders are connected in series forming an electrical resistance to which power is applied. The heat is released through Joule heating, $P_{e}=R I^{2}$, where each cylinder resistance is, $R=\rho L / A$, where $\rho$ is the electrical resistivity of the material, $L$ the length of the cylinder and $A$ the cross-sectional area. The cross-section area along the resistance path in the STAR heat exchanger is tailored to control dissipation and limit the highest propellant stagnation temperature to the inner cylinder and in close proximity to the nozzle inlet.

The prototype presented is constructed of 316L stainless steel (SS), which limits the achievable gas temperature to approximately $930 \mathrm{~K}$ and is intended as a proof of concept validation of the operation and performance. The subsequent development of the STAR heat exchanger uses refractory metals, including tantalum [7] and tungsten, in an engineering model able to reach the target ISP with xenon propellant at an operating temperature in the region of 3000K. In [8], high-temperature candidate materials for the engineering model are discussed, highlighting that the target ISP is achievable.

The purpose of this paper is to provide the validation of a concept heat exchanger produced via SLM and to analyse the experimental data in comparison with 2D and 3D numerical models.

${ }^{*}$ Corresponding author.

E-mail address: f.romei@soton.ac.uk 


\begin{tabular}{|c|c|c|c|}
\hline \multicolumn{2}{|c|}{ Acronyms } & \multirow{2}{*}{$\begin{array}{l}p \\
P\end{array}$} & \multirow{2}{*}{$\begin{array}{l}\text { Pressure [bar] } \\
\text { Power [W] }\end{array}$} \\
\hline EB & Electron Bean welding & & \\
\hline EDM & Electric Discharge Machining & $q$ & Heat flux $\left[\mathrm{W} / \mathrm{m}^{2}\right]$ \\
\hline f.f.t. & Feedthrough & $R$ & Heater resistance $[\mathrm{m} \Omega]$ \\
\hline FGSE & Fluidic Ground Support Equipment & $t$ & Time $[\mathrm{s}]$ \\
\hline MFC & Mass Flow Controller & $T$ & Temperature $[\mathrm{K}]$ \\
\hline PC & Pressure Controller & $\mathrm{TC}_{\mathrm{j}}$ & j-th measured temperature $[\mathrm{K}]$ \\
\hline RMS & Root mean square & $v$ & Velocity $[\mathrm{m} / \mathrm{s}]$ \\
\hline SLM & Selective Laser Melting & $V$ & Thruster heater potential [V] \\
\hline SS & Stainless Steel & $\gamma$ & Ratio of specific heats [-] \\
\hline STAR & Super-high Temperature Additive Resistojet & $\varepsilon$ & Surface emissivity [-] \\
\hline TIG & Tungsten Inert Gas welding & $\eta$ & Efficiency [-] \\
\hline \multicolumn{2}{|c|}{ Symbols } & $\rho$ & Electrical resistivity $[\Omega \mathrm{m}]$ \\
\hline A & Section Area $\left[\mathrm{m}^{2}\right]$ & \multicolumn{2}{|c|}{ Subscripts } \\
\hline$c_{p}$ & Specific heat capacity at constant p. $[\mathrm{J} /(\mathrm{kgK})]$ & 0 & stagnation $\left(p_{0}, T_{0}\right)$ \\
\hline$f$ & Refinement parameter [-] & e & electric $\left(P_{e}\right)$ or nozzle exit $\left(A_{e}, M_{e}, v_{e}\right)$ \\
\hline$F$ & Thrust $[\mathrm{mN}]$ & $\mathrm{h}$ & heater $\left(t_{h}, \eta_{h}\right)$ \\
\hline I & Electrical current $[\mathrm{A}]$ & in & inlet $\left(p_{0, \text { in }}, T_{0, \text { in }}\right)$ \\
\hline$I_{s p}$ & Specific impulse [s] & $\mathrm{j}$ & jet $\left(P_{j}\right)$ \\
\hline $\boldsymbol{J}$ & Current density $\left[\mathrm{A} / \mathrm{m}^{2}\right]$ & $\mathrm{n}$ & nozzle $\left(\eta_{n}\right)$ \\
\hline$M$ & Mach number [-] & $\mathrm{t}$ & throat $\left(A_{t}, M_{t}, R e_{t}\right)$ \\
\hline$\dot{m}$ & Mass flow rate $[\mathrm{mg} / \mathrm{s}]$ & ts & thruster $\left(P_{t s}, \eta_{t s}\right)$ \\
\hline$n$ & Unit normal vector [-] & vc & vacuum chamber $\left(p_{v c}\right)$ \\
\hline
\end{tabular}

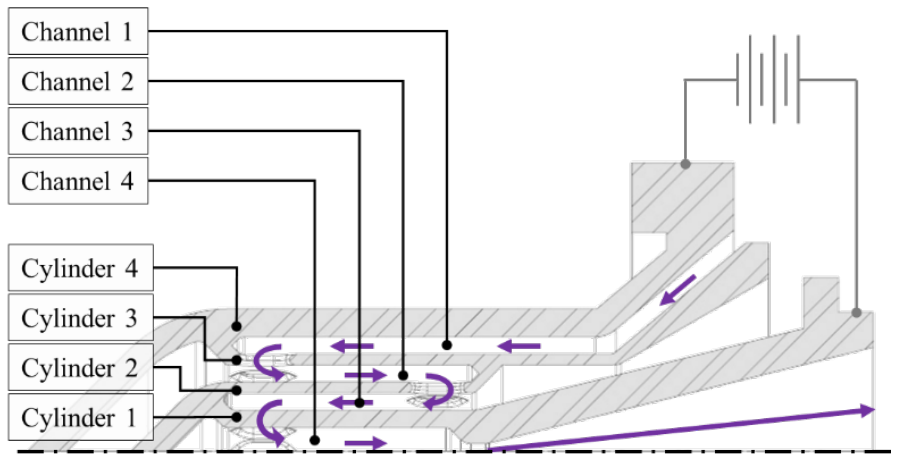

Fig. 1. Axisymmetric schematics of the monolithic heat exchanger concept. The propellant flow path (purple) and the electrical interface are shown [1 column width].

\section{Experimental Test Setups and Computational Models}

In this section, the assembly details of the first prototype (STAR-0), test setup and thrust measurement system are described. Two prototype models are tested, in particular, STAR-0-A is subjected to dry electrothermal tests, while STAR-0-B is subjected to wet performance tests with argon propellant. The 3D and 2D computational models used for performance verification are also introduced such that they can be later used to gain insight into the performance data.

\subsection{STAR-0 High-Temperature Resistojet Assembly Description}

Fig. 2 depicts the STAR-0 design and shows a radiograph of the STAR-0-B assembly, while Table 1 lists the key components with respective manufacturing processes and nominal weight. The total weight of the assembly, not including fasteners, is approximately $190 \mathrm{~g}$. The SLM components are printed on a Concept Laser M2 Cusing SLM machine, using the process parameters 
listed in [4]. Once printed, the excess powder is removed and the components are cut from the build plate by electro-discharge machining (EDM) wire-cut. Both the heat exchanger and the thruster inflow require minimal CNC post-manufacturing. The nozzle is drilled to obtain the nominal throat diameter [4] and the assembly includes two electron beam (EB) welds to join the heat exchanger with the thruster inflow and thruster casing. The EB welds ensure both electrical continuity and hermetic sealing. In addition, two fusion TIG welds are performed to join the thruster inflow with a support and a 1/8 in. SS propellant supply pipe. The cold propellant enters the thruster through the supply pipe, passing through the thruster inflow component and following an annular path, which envelopes the thruster body with relatively cold propellant up to a nozzle spacer. The latter blocks the path of propellant and ensures that the majority of the flow moves towards channel (1) while maintaining electrical isolation between the walls. The propellant continues flowing through the four regenerative recuperation channels, finally arriving at a converging-diverging nozzle. A ceramic collar serves for both leak tightness and electric isolation. Electrical power is supplied through positive and negative ring tab terminals positioned at the casing and at the support respectively, with the current flowing through the metallic body. The STAR0 has four concentric cylinders with a nominal thickness of $300 \mu \mathrm{m}, 150 \mu \mathrm{m}, 150 \mu \mathrm{m}$ and $300 \mu \mathrm{m}$, where the innermost cylinder is $22 \mathrm{~mm}$ long. The design of the connectors and micro-fluidic channels, allowing the movement of flow between cylinders, are reported in [4]. The gaps between the cylinders make four channels measured from the innermost respectively as $0.6 \mathrm{~mm}, 0.8 \mathrm{~mm}$ and $1 \mathrm{~mm}$. The nozzle has a nominal throat diameter of $0.42 \mathrm{~mm}$, a half-angle divergence of $14^{\circ}$, and inlet and outlet area ratios with respect to the throat of 15 and 211 respectively. The portion of room temperature resistance from the innermost cylinder to the outermost is $40 \%, 31 \%, 21 \%$ and $8 \%$ respectively of the total heat exchanger resistance. It has to be noted that the ceramic collar gasket and the metallic flanges were insufficient to give leak tightness and are therefore bound with epoxy resin. The EB and TIG welds performed as expected both in terms of leak tightness and electrical continuity.

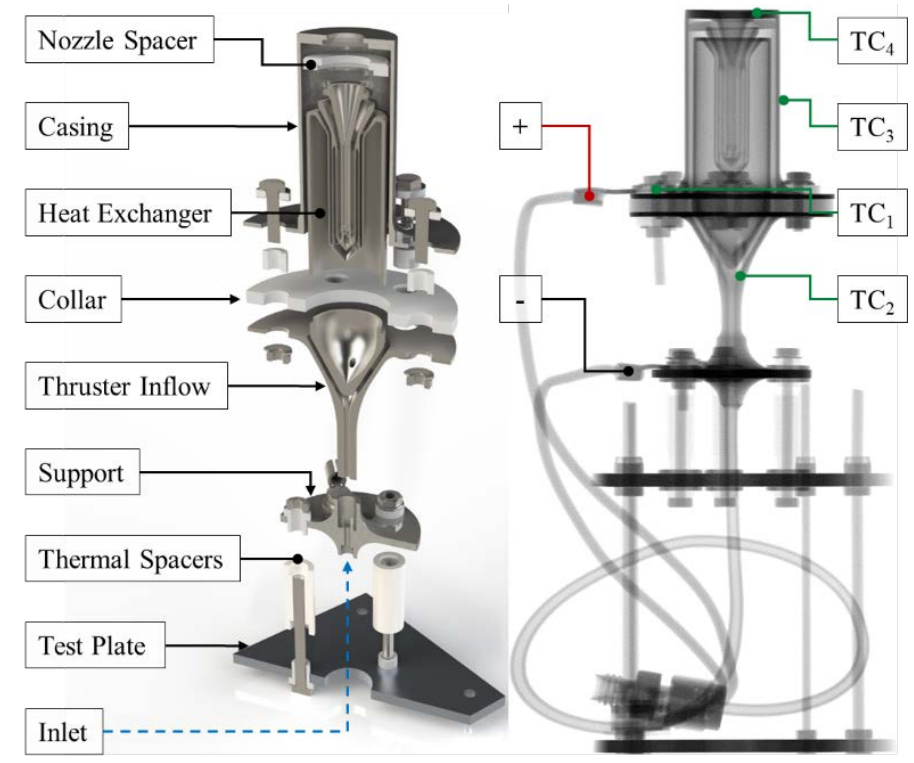

Fig. 2. Half-section exploded view of STAR-0 (left) and scatter-free radiograph of the STAR-0-B assembly with thermocouples and ring terminal positions (right) [1 column width].

Table 1. Main components of the STAR-0 resistojet.

\begin{tabular}{lccc}
\hline \multicolumn{1}{c}{ Component } & Material & Manufacturing process & $\begin{array}{c}\text { Nominal } \\
\text { Weight [g] }\end{array}$ \\
\hline Heat Exchanger & 316L SS & SLM + CNC post & 20.6 \\
Thruster Inflow & 316L SS & SLM + CNC post & 28.3 \\
Casing & 316L SS & CNC & 36.2 \\
Support & 316L SS & CNC & 20.4 \\
Collar & Macor & CNC & 11.2 \\
Nozzle Spacer & Shapal & CNC & 1.3 \\
Ceramic thermal spacers & Macor & CNC & 24.8 \\
\hline
\end{tabular}

\subsection{Computational Models}

The laminar flow modelling equations are Eq.(1), the mass conservation, Eq.(2), conservation of momentum, and Eq.(3), conservation of energy in terms of temperature, where $\rho$ is the fluid density, $\mathbf{u}$ is the velocity vector (with axial and radial components $w$ and $u$, respectively), $C_{p}$ is the specific heat capacity at constant pressure and $k$ the thermal conductivity. The resistive 
heating is modelled through Eq.(4), where $Q_{e}$ is the resistive heating due to the electric current, $\mathbf{J}$ the current density and $\mathbf{E}$ the electric field.

$$
\begin{gathered}
\frac{\partial \rho}{\partial t}+\nabla \cdot(\rho \mathbf{u})=0 \\
\rho \frac{\partial \mathbf{u}}{\partial t}+\rho(\mathbf{u} \cdot \nabla) \mathbf{u}=\nabla \cdot\left[-p \mathbf{I}+\mu\left[\nabla \mathbf{u}+(\nabla \mathbf{u})^{T}\right]-\frac{2}{3} \mu(\nabla \cdot \mathbf{u}) \mathbf{I}\right] \\
\rho C_{p} \frac{\partial T}{\partial t}+\rho C_{p} \mathbf{u} \cdot \nabla T=\nabla \cdot(k \nabla T)+Q_{e} \\
Q_{e}=\mathbf{J} \cdot \mathbf{E}
\end{gathered}
$$

A 3D sector-symmetric electrothermal model developed in COMSOL Multiphysics, which uses Eq.(3) and Eq.(4), is used to evaluate the performance of the resistojet and serves as a validated model for future design iterations. The computed 3D sector is $1 / 6^{\text {th }}$ of the total geometry, therefore the current applied to the terminal boundary condition $(\mathrm{BC})$ is $1 / 6^{\text {th }}$ of the experimental value. The materials, 316L (labelled [solid, polished]) and Macor (labelled [solid]), are selected from the materials library, while the thermal properties of Shapal are added manually from datasheets. The electrical resistivity of 316L is extrapolated from $873 \mathrm{~K}$ to $1300 \mathrm{~K}$ using an interpolating function. The materials properties used for Macor and Shapal are the thermal conductivity, density, surface emissivity and heat capacity, while for 316L electrical conductivity is also modelled.

A computational grid convergence study on the stationary solution is performed as a function of a refinement parameter, $f$. The surface emissivity of SLM 316L is fixed to 0.6. The selected parameter value for simulations is $f=3$ (corresponding to 63,822 elements), which produces a solution with a relative error $<10^{-3}$ with respect to $f=10$ (198,667 elements). The error is defined as the maximum relative error with respect to the finest mesh amongst the four probed temperatures and the electrical resistance. BCs of symmetry are applied to the sector side boundaries for the heat as $\boldsymbol{n} \cdot \boldsymbol{q}=0$, where $\boldsymbol{n}$ is the unit vector normal to the surface and $\boldsymbol{q}$ the heat flux, and for the surface-to-surface radiation. An electric insulation BC is applied to the same boundaries as $\boldsymbol{n} \cdot \boldsymbol{J}=0$, where $\boldsymbol{J}$ is the current density. In the model, we assume that all thermal contacts are ideal. The radiograph depicts that the nozzle spacer is not in full contact with the top part of the casing as in the design (Fig. 2). The difference in simulation results is large between the two cases of assuming full contact and of introducing a gap between the two components. As highlighted in Table 2, the RMS, calculated on the relative errors of the measured quantities, is larger when assuming that the nozzle spacer is in ideal contact with the casing. With a gap of $0.5 \mathrm{~mm}$, the root-mean-square (RMS) error decreases from 0.267 to 0.167 . The solution has also been found particularly sensitive to the assumptions on the emissivity of the SLM surfaces. where $\varepsilon_{1}$ is the emissivity of 316L SS found in [9] and $\varepsilon_{2}$ the emissivity of polished 316 SS found in [10]. With the third row assumptions, the RMS error reaches a value of 0.097, which is considered sufficient for discussion on the model results.

Table 2. Simulation over experimental error with different assumptions.

\begin{tabular}{ccc|ccccccc}
\hline \multicolumn{2}{c|}{ Assumptions } & \multicolumn{7}{c}{ Results } \\
\hline Geometry & $\varepsilon_{S L M}$ & $\varepsilon_{C N C}$ & $\tilde{T}_{1}$ & $\tilde{T}_{2}$ & $\tilde{T}_{3}$ & $\tilde{T}_{4}$ & $\tilde{R}_{t s}$ & RMS err. \\
\hline Spacer with contact & 0.6 & $\varepsilon_{1}$ & 0.55 & 1.32 & 1.02 & 1.20 & 1.08 & 0.267 \\
Spacer with gap & 0.6 & $\varepsilon_{1}$ & 0.80 & 0.84 & 0.93 & 0.75 & 0.91 & 0.167 \\
Spacer with gap & $\varepsilon_{2}$ & $\varepsilon_{1}$ & 0.99 & 0.97 & 1.19 & 0.95 & 0.92 & 0.097 \\
\hline
\end{tabular}

A number of additional factors can influence the solution, including the unknown thermal properties of the SLM material, for which the standard properties have been used. For example, it has been shown that there is a correlation between surface roughness and emissivity, however, this is expected to be within $\pm 5 \%$ change as discussed in [11]. In addition, the porosity of the SLM components can produce a higher electrical resistivity [12]. Moreover, the two EB welds could also produce a thermal contact resistance and a change in the heat fluxes between the components. Despite the modest agreement of the model with the experimental measurements, the solution reveals an insight on the temperature distribution inside the heat exchanger, highlighting the location of maximum structural temperature and increase of temperature inside the channels. The overall agreement of the simulation with experiments expressed as the RMS is of $<10 \%$.

In addition to the 3D model, a 2D axisymmetric model described in [8], which uses Eq.(1) to Eq.(4), is used to evaluate the electrothermal-fluidic characteristic of the STAR-0-B prototype and is presented in the discussion. The parametrised geometry has been modified to reflect the prototype geometry and the assumptions described in the last row of Table 2 are also used. The RMS total errors on the five selected variables are 0.121 and 0.097 for the $2 \mathrm{D}$ and 3D models respectively. For this reason, the 2D model can be used confidently for the evaluation of the electrothermal-fluidic solution of the thruster. The thrust exerted by the nozzle is calculated using Eq.(5), where $r_{e}$ is the nozzle exit section radius. 


$$
F=\int_{0}^{2 \pi} \int_{0}^{r_{e}}\left(\rho w^{2}+p\right) d r d \vartheta
$$

For all time-dependant simulations, backward differentiation formulas (BDF) with a maximum order of accuracy of two are employed. The time stepping is free and the relative tolerance is of 0.005 . Solutions are provided at the steps taken by the solver.

\subsection{Experimental Test Facility}

The tests presented in this paper were conducted at the David Fearn Electric Propulsion Laboratory of the University of Southampton. The cryogenic vacuum facility is composed of a $4 \mathrm{~m}$ long by $2 \mathrm{~m}$ diameter main test chamber and an additional loading chamber separated by at $50 \mathrm{~cm}$ gate valve. The pumping equipment consists of two cryopumps, three turbomolecular pumps and two backing pumps. The facility can reach an ultimate vacuum of $<9 \times 10^{-8} \mathrm{mbar}$ with operation $<5.0 \times 10^{-5} \mathrm{mbar}$ with $<28 \mathrm{sccm}$ xenon ( 2.8 mg/s) Effective xenon pumping speed at $5 \times 10^{-5}$ mbar with a nominal pumping speed of $27790 \mathrm{l} / \mathrm{s}$. The test setup used for the wet performance evaluation of the STAR-0-B prototype is shown in Fig. 3. The 1/8 in. SS inflow pipe and the electrical terminals are fixed on a second aluminium interface plate positioned below the thruster. Argon is supplied to the thruster via a 1/8 in. PFA flexible pipe, which is fixed to a levelled aluminium interface on the opposite end of the thruster to keep any flexural force on the balance to a minimum.

Thrust is measured with a Mettler Toledo WMS404C-L/01 weighing module, with the resistojet mounted vertically. Before each test, an in-air adjustment routine was performed using the APW-Link software. This involves compensation in two measurement points, the zero point (no weight) and a test mass of $300 \mathrm{~g}$, consisting of a single E2 calibration weight. The balance was then used for the vacuum tests without performing further calibration. The error on the thrust measurement is based on the manufacturer datasheet in a worst-case scenario, using the limit values rather than the nominal accuracy. In particular, it is calculated as the sum of the repeatability $(0.1 \mathrm{mg})$, linearity deviation $(0.4 \mathrm{mg})$, eccentric load deviation $(1 \mathrm{mg})$, sensitivity offset (2 mg), sensitivity temperature drift $\left(0.00015 \% /{ }^{\circ} \mathrm{C} \times R_{n t}\right)$ and sensitivity stability $\left(0.00025 \% /{ }^{\circ} \mathrm{C} \times R_{n t} \times 20^{\circ} \mathrm{C}\right)$, where $R_{n t}=400 \mathrm{~g}$ is the maximum net weight and $a=1$ the year from last calibration. The resulting uncertainty is of $\pm 0.16 \mathrm{mN}$. It must be stressed that the proposed measured uncertainty does not include the effect of vacuum, of the stiffness of the 1/8 in. PFA propellant pipe, of the environmental vibration disturbances and of the interaction between melted Ga and the copper rods. However, after each one of the wet firing tests, the thrust measurement recovered within the readability of the instrument. For these reasons, the thrust balance uncertainty can differ from the proposed value. Future work will aim to perform in-situ measurements with calibration weights to evaluate experimentally the total accuracy of the system.

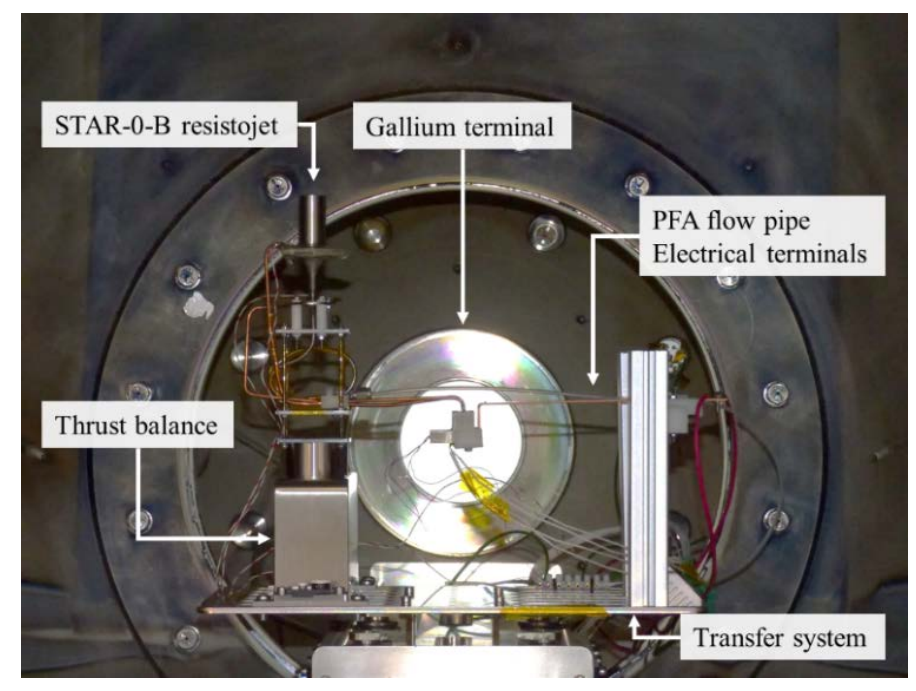

Fig. 3. STAR-0-B test rig located into the main test chamber via the transfer system [1 column width].

During an initial test hot fire measurements experienced a significant thermal drift [8], attributed to the thermal expansion of the thruster terminal wires. A drift-free thrust measurement system was developed and described in [13]. The electrical terminal consists of two SS baths, each one equipped with a 10W cartridge heater (Fig. 4, left). Each bath contains approximately 8 grams of gallium, which is maintained in liquid state (Fig. 4, centre) by heating to $35^{\circ} \mathrm{C}$ with a hysteresis cycle of $\pm 0.5^{\circ} \mathrm{C}$. Two $1 / 8$ in. copper rods are connected to the thruster positive and negative terminal and positioned in liquid Ga. During hot-fire operation, the terminal rods are unconstrained under thermal expansion and do not influence thrust measurement. 

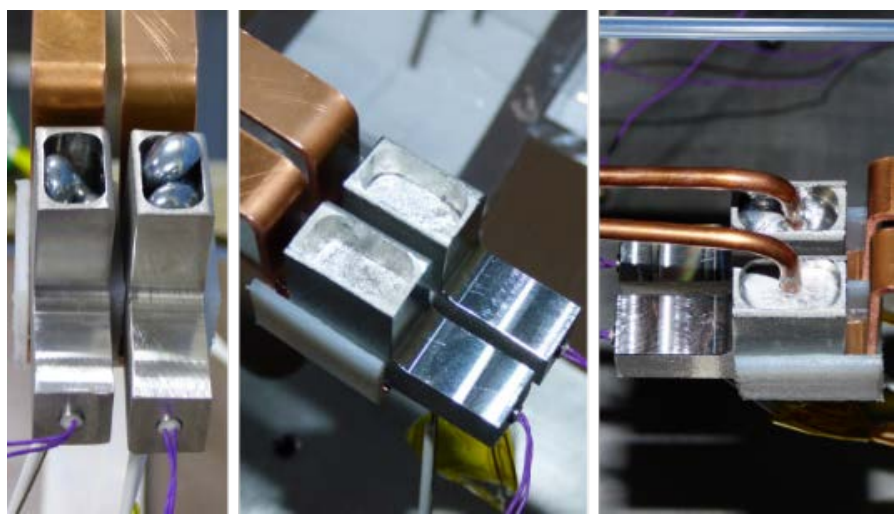

Fig. 4. Gallium electrical terminal overview: intact gallium shots at ambient temperature (left), melted gallium (centre) and resistojet terminals submerged into the liquid metal (right) [1 column width].

\subsection{Experimental Apparatus}

Table 3 lists the equipment used for the tests, as well as the manufacturer, type of measurement, range, nominal accuracy and measurement accuracy of each instrument. A Bronkhorst based Fluidic Ground Support Equipment (FGSE) provides either flow rate or pressure controlled argon supply to the thruster. The mass flow meter and a forward pressure controller are connected in series, with the pressure controller fully opened and used to read out the pressure at controlled mass flow rates. The two controllers are connected to an E-8501-R-20 digital readout and control unit. A high-current power supply, the controller unit of the FGSE, the DAQ system, the thrust balance and the oscilloscope are all connected to a workstation as shown in Fig. 5 and controlled using a LabVIEW interface, which uses the respective VISA drivers. In addition to data acquisition, the interface is used to control the MFC and PC, the power supply output and the heaters used for the Ga terminal, as well to write the reset and calibration commands to the thrust balance.

The high-current power supply provides current limited DC power to the resistojet terminals. Two 10 AWG wires with 105 copper strands connect the power supply to two different KF flanged feedthroughs with a single copper conductor of $2.4 \mathrm{~mm}$ diameter. A NI cDAQ-9188XT with a NI 9213 thermocouple module and a NI 9264 analogue output module is used the read the thermocouples values and to control the heaters of the Ga-terminal. Each heater of the Ga-terminal is activated with an Omega SSR240 solid state relay, which is controlled via analogue outputs from the DAQ. The thrust balance is connected from the feedthrough to a terminal block, which supplies $30 \mathrm{VDC}$ and the communication interface. An oscilloscope was used to measure the voltage at the thruster terminals, $V$, at the feedthrough via a $250 \mathrm{MHz}$ passive probe. Finally, a 4-wire milli-Ohm meter was used to check the resistojet cold resistance at ambient conditions.

Table 3. Summary of instrumentation used for the tests with measurement accuracy.

\begin{tabular}{lcllll}
\hline \multicolumn{1}{c}{ Instrument } & Sym. & \multicolumn{1}{c}{ Range used } & \multicolumn{1}{c}{ Manufacturer } & \multicolumn{1}{c}{ Type } & \multicolumn{1}{c}{ Accuracy } \\
\hline Thrust balance & $F$ & $0-400 \mathrm{~g}$ & Mattler Toledo & WMS404C & 0.1 mg readability \\
MFC & $\dot{m}$ & $0.06-3 \mathrm{ln} / \mathrm{min}(\mathrm{Xe})$ & Bronkhorst & F-201CV-5K0 & $\pm 0.5 \% \mathrm{RD}$ plus $\pm 0.1 \% \mathrm{FS}$ \\
PC & $p_{P C}$ & $0.12-6 \mathrm{bar}(\mathrm{g})$ & Bronkhorst & P-602CV-21KA & $\pm 0.5 \% \mathrm{FS}$ \\
Power supply & $I$ & $0-157.5 \mathrm{~A}$ & Kikusui & PWX1500L & $\pm(0.5 \%$ of set $+0.1 \%$ of rating) \\
Oscilloscope & $V$ & $1 \mathrm{~V}, 2.5 \mathrm{~V}, 5 \mathrm{~V}$ & LeCroy & Wavesurfer $3024 \pm(1.0 \%$ of offset value $+3 \% \mathrm{FS}+1 \mathrm{mV})$ \\
DAQ TCs mod. & $\mathrm{TC}_{1-4}$ & $-260-1260^{\circ} \mathrm{C}$ & National Instrument & NI 9213 & $\pm 0.25^{\circ} \mathrm{C}$ \\
& & $1 \mathrm{~m} \Omega-1.999 \Omega$ & Rhopoint Components Ltd & $\mathrm{M} 210$ & $\pm(0.1 \%$ of range $+1 \mathrm{~m} \Omega$ zero offset $)$ \\
\hline
\end{tabular}




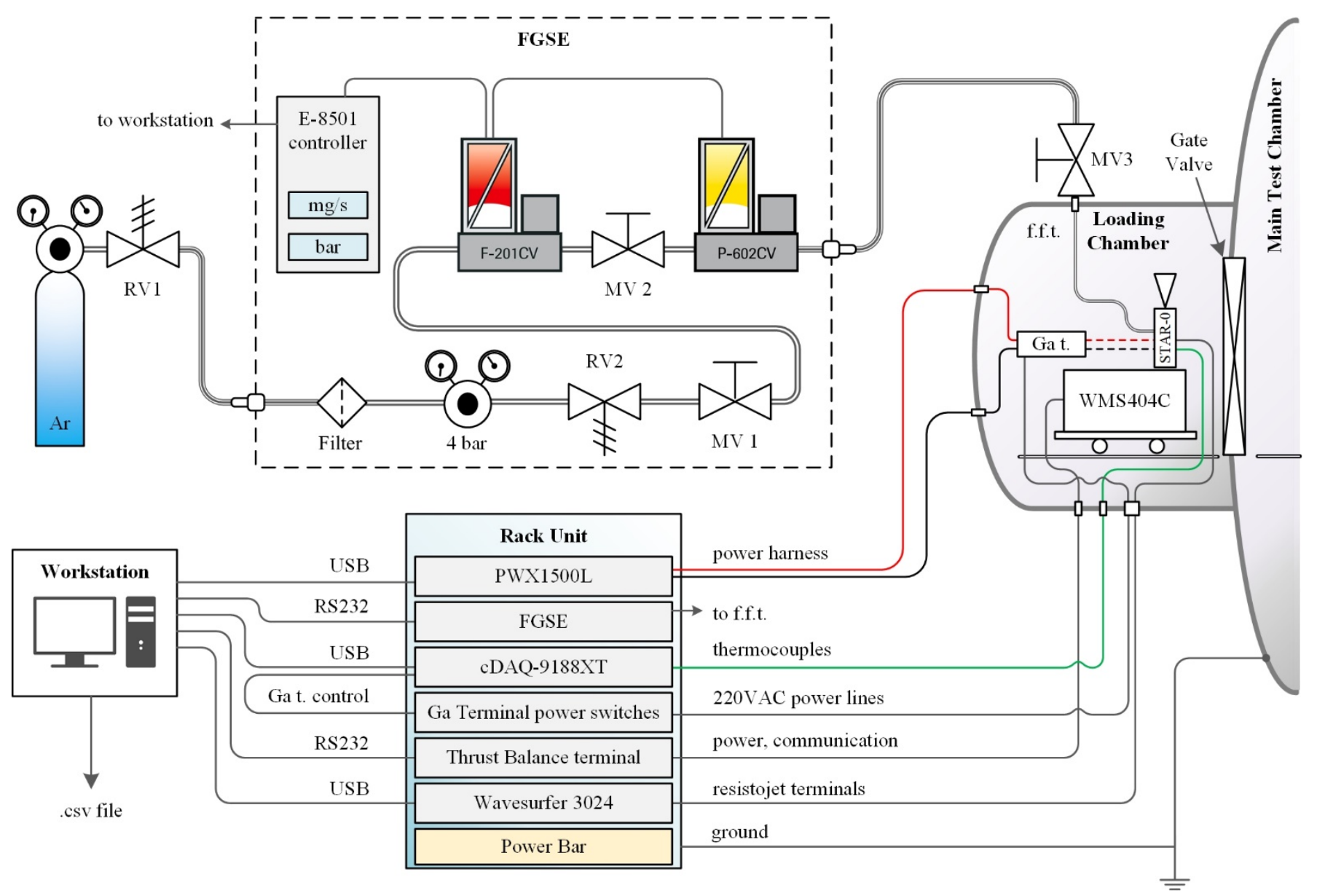

Fig. 5. Schematics of test setup [2 column width].

\subsection{Methodology}

Two identical prototypes, STAR-0-A and STAR-0-B, are tested in two different ways. The first is used to evaluate the electrothermal dry characteristics over a long heating time. The second prototype is used for evaluating the wet performance of the thruster, deriving the maximum ISP for a range of electrical currents and with two different flow rates of Ar gas. The direct measurements taken in the test campaign were the following (Table 3): thrust, $F$, mass flow rate, $\dot{m}$, pressure measured at the pressure controller, $p_{P C}$, current read from the power supply, $I$, voltage measured at the resistojet terminals, $V$, temperatures, $T_{j}$. The mass flow controller was calibrated for Xe, therefore a conversion factor $x$ was used to derive the Ar flow rate in accordance with the Bronkhorst conversion data for the specific MFC [14], where $\dot{m}(\mathrm{Ar})=x \dot{m}(\mathrm{Xe})$. In particular, at the two test mass flow rates M1 and M2, $x=0.3164$ and $x=0.3222$, respectively. The uncertainty of direct measurements, summarised in shown in Table 3, is calculated using the manufactures datasheets.

The STAR-0-A prototype is subjected to a range of electrical input currents without propellant flow. Four temperatures, TC 1 to $\mathrm{TC}_{4}$, are measured at selected points of the thruster body through k-type thermocouples as shown in Fig. 2. With reference to Fig. 5 , the dry test only makes use of the electrical test setup. In addition, neither the gallium terminal nor the thrust balance is used. The dry tests are performed in the loading chamber and in vacuum conditions. At the lower current case, the oscilloscope is set to 100 $\mathrm{mV} / \mathrm{div}$ ( $\pm 0.5 \mathrm{~V}$ of range), while for the remaining three tests it is set to $500 \mathrm{mV} / \mathrm{div}$ ( $\pm 2.5 \mathrm{~V}$ of range). The voltage measurement is time-averaged over four sweeps, with a sweep being a $100 \mathrm{~ms}$ time window at $100 \mathrm{kS} / \mathrm{s}$. The heating time is of $2500 \mathrm{~s}$ in all four cases. The junctions of $\mathrm{TC}_{1}$ and $\mathrm{TC}_{2}$ are electrically insulated from the resistojet body using Kapton tape. $\mathrm{TC}_{3}$ and $\mathrm{TC}_{4}$ are spot welded to the respective reference points.

Wet tests are performed at constant volumetric flow rate using Ar gas. Measurements taken in hot gas tests include thrust, the forward pressure at the PC, the mass flow rate of Ar at the MFC. In addition, the resistojet terminal voltage was measured via an oscilloscope. For the tests M1-1 to M1-3 and M2-1 to M2-3 the measurement was set to $100 \mathrm{mV} / \mathrm{div}$ ( $\pm 0.5 \mathrm{~V}$ of range) with an offset of $300 \mathrm{mV}$. For the remaining tests, the measurement was set $200 \mathrm{mV} /$ div ( $\pm 1 \mathrm{~V}$ of range) with an offset of $0.2 \mathrm{~V}$. The voltage measurement was time-averaged over four sweeps, with a sweep being a $100 \mathrm{~ms}$ time window at $100 \mathrm{kS} / \mathrm{s}$. Tests are performed in the main test chamber at a base pressure of $2 \times 10^{-6}$ mbar with cryopumps. The testing method consisted of the following process steps:

1. The current is set to $3 \mathrm{~A}$, which corresponds to a negligible power dissipation of $0.3 \mathrm{~W}$ while permitting the monitoring of the electrical resistance;

2. The MFC is commanded to the desired mass flow rate; 
3. When the thrust reaches a steady value in cold mode and the chamber base pressure stabilises, the thruster is switched to hot fire mode, by increasing the electrical current to the prescribed setpoint;

4. After a given time, the current is decreased to 3A then the flow controller is closed;

The STAR-0-B prototype was tested with two flow rates, corresponding to cases M1 and M2 respectively, and the heater was switched on in current limited mode at four increasing electrical currents. The electrical currents were selected to achieve a maximum expected power input of $10 \mathrm{~W}, 15 \mathrm{~W}, 20 \mathrm{~W}$ and $25 \mathrm{~W}$, assuming a final hot resistance of $45 \mathrm{~m} \Omega$.

\section{Results}

Table 4 shows the summary of results of the wet tests in the two cases M1 and M2, where the parameters are evaluated at the maximum thrust level achieved with the electrothermal enhancement in the heating time $t_{h}$ for each case. Direct measurements are indicated with a dashed line, while a formula for the reaming quantities is shown in the reference column. Fig. 6 depicts the transient of the thrust for the test cases analysed.

3.

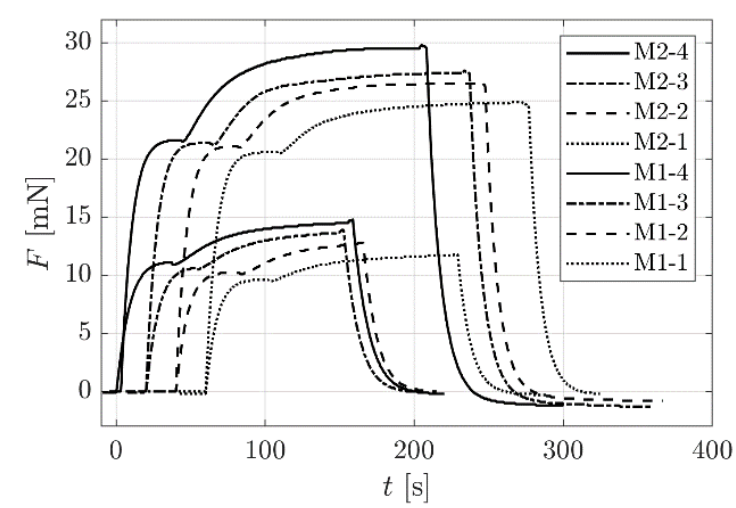

Fig. 6. Thrust transient of the M1 and M2 test series. Each start front of same test currents is separated by 20 seconds for presentation clarity. [1 column width].

From the direct measurements listed in Table 3, the derived parameters of interest are the specific impulse, $I_{s p}$, given by Eq.(6), the electrical power of the thruster, $P_{e}$, the total thruster power input, $P_{t s}$, and the thruster efficiency, $\eta_{t s}$, given by Eq.(7). $P_{0, i n}=$ $c_{p} T_{0, \text { in }}$ is the inlet thermal energy of the cold gas and $P_{0}=c_{p} T_{0}$ is the stagnation energy of the gas at the nozzle inlet.

$$
\begin{gathered}
I_{s p}=\frac{F}{\dot{m} g_{0}} \\
\eta_{t s}=\eta_{h} \eta_{n}=\frac{P_{0}}{P_{t s}} \frac{P_{j}}{P_{0}}=\frac{\text { kinetic power }}{\text { total inlet power }}=\frac{F^{2} / 2 \dot{m}}{P_{0, \text { in }}+P_{e}}
\end{gathered}
$$

The relative uncertainties of the derived parameters are calculated assuming that the quantities analysed have errors which are uncorrelated and random. As an example, Eq.(8) represents the relative error of the thruster total efficiency.

$$
\frac{\delta \eta_{t s}}{\left|\eta_{t s}\right|}=\sqrt{2\left(\frac{\delta F}{F}\right)^{2}+\left(\frac{\delta \dot{m}}{\dot{m}}\right)^{2}+\left(\frac{\delta P_{t s}}{P_{t s}}\right)^{2}}
$$


Table 4. Summary of M1 and M2 tests with quantities evaluated at the maximum measured thrust (2 columns).

\begin{tabular}{|c|c|c|c|c|c|c|c|c|c|c|c|c|}
\hline \multirow{2}{*}{ Sym. } & \multirow{2}{*}{ Unit } & \multirow{2}{*}{ Ref. } & \multicolumn{10}{|c|}{ Test number } \\
\hline & & & M1-0 & M1-1 & M1-2 & M1-3 & M1-4 & M2-0 & M2-1 & M2-2 & M2-3 & M2-4 \\
\hline$\dot{m}$ & $\mathrm{mg} / \mathrm{s}$ & - & \multicolumn{5}{|c|}{$18.84 \pm 0.28$} & \multicolumn{5}{|c|}{$38.44 \pm 0.38$} \\
\hline$F^{*}$ & $\mathrm{mN}$ & - & 9.7 & 11.8 & 12.8 & 13.9 & 14.8 & 20.3 & 24.9 & 26.6 & 27.6 & 29.8 \\
\hline \multirow{2}{*}{$I_{s p}$} & \multirow{2}{*}{ s } & \multirow{2}{*}{ Eq.(6) } & 52.51 & 63.87 & 69.29 & 75.24 & 80.11 & 53.86 & 66.06 & 70.57 & 73.22 & 79.06 \\
\hline & & & \pm 1.17 & \pm 1.30 & \pm 1.36 & \pm 1.43 & \pm 1.49 & \pm 0.68 & \pm 0.78 & \pm 0.82 & \pm 0.84 & \pm 0.89 \\
\hline \multirow{2}{*}{$P_{e}$} & \multirow{2}{*}{ W } & \multirow{2}{*}{$V \cdot I$} & 0.300 & 8.129 & 12.751 & 17.880 & 23.560 & 0.300 & 7.943 & 12.422 & 17.335 & 22.648 \\
\hline & & & \pm 0.060 & \pm 0.310 & \pm 0.389 & \pm 0.731 & \pm 0.8260 & \pm 0.060 & \pm 0.309 & \pm 0.387 & \pm 0.730 & \pm 0.823 \\
\hline \multirow{2}{*}{$\eta_{t s}$} & \multirow{2}{*}{$\%$} & \multirow{2}{*}{ Eq. (7) } & 77.4 & 33.4 & 27.7 & 24.6 & 21.9 & 85.5 & 58.0 & 50.0 & 42.5 & 40.4 \\
\hline & & & \pm 3.7 & \pm 1.2 & \pm 0.9 & \pm 1.0 & \pm 0.8 & \pm 3.3 & \pm 1.5 & \pm 1.2 & \pm 1.4 & \pm 1.3 \\
\hline \multirow{2}{*}{$R$} & \multirow{2}{*}{$\mathrm{m} \Omega$} & \multirow{2}{*}{$V / I$} & 33.3 & 36.5 & 38.0 & 40.1 & 42.3 & 33.3 & 35.6 & 37.0 & 38.9 & 40.6 \\
\hline & & & \pm 6.6 & \pm 1.4 & \pm 1.2 & \pm 1.6 & \pm 1.5 & \pm 6.6 & \pm 1.4 & \pm 1.2 & \pm 1.6 & \pm 1.5 \\
\hline \multirow{2}{*}{$V$} & \multirow{2}{*}{ V } & \multirow{2}{*}{-} & 0.100 & 0.545 & 0.696 & 0.847 & 0.998 & 0.100 & 0.532 & 0.678 & 0.821 & 0.959 \\
\hline & & & \pm 0.019 & \pm 0.019 & \pm 0.019 & \pm 0.033 & \pm 0.033 & \pm 0.019 & \pm 0.019 & \pm 0.019 & \pm 0.033 & \pm 0.033 \\
\hline \multirow{2}{*}{$I$} & \multirow{2}{*}{ A } & \multirow{2}{*}{-} & 3.000 & 14.924 & 18.318 & 21.112 & 23.610 & 3.000 & 14.930 & 18.321 & 21.115 & 23.616 \\
\hline & & & \pm 0.173 & \pm 0.232 & \pm 0.249 & \pm 0.263 & \pm 0.276 & \pm 0.173 & \pm 0.232 & \pm 0.249 & \pm 0.263 & \pm 0.276 \\
\hline \multirow{2}{*}{$P_{t s}$} & \multirow{2}{*}{ W } & \multirow{2}{*}{$P_{0, i n}+P_{e}$} & 3.227 & 11.056 & 15.678 & 20.807 & 26.488 & 6.272 & 13.915 & 18.394 & 23.308 & 28.620 \\
\hline & & & \pm 0.124 & \pm 0.310 & \pm 0.389 & \pm 0.731 & \pm 0.833 & \pm 0.220 & \pm 0.309 & \pm 0.387 & \pm 0.730 & \pm 0.823 \\
\hline$p_{P C}^{\dagger}$ & bar & - & 0.65 & 0.81 & 0.87 & 0.94 & 1.01 & 1.28 & 1.55 & 1.65 & 1.71 & 1.85 \\
\hline$t_{h}$ & $\mathrm{~s}$ & - & 0 & 123.9 & 78.9 & 97.3 & 118.4 & 0 & 160.7 & 160.4 & 167.8 & 159.6 \\
\hline
\end{tabular}

${ }^{*}$ Uncertainty of $\pm 0.16 \mathrm{mN}$

4.

$\dagger$ Uncertainty of \pm 0.02 bar

\section{Discussion}

4.1 Dry Firing Performance

The cold resistance measured at the thruster terminals at ambient conditions with the 4-wire Ohm-meter was of $30 \pm 3 \mathrm{~m} \Omega$ pre and post-test. The corresponding model resistance is of $27.6 \mathrm{~m} \Omega$. Fig. 7 depicts the temperature measurements (solid lines) and the simulated values (markers) in the test time window of $2500 \mathrm{~s}$ at the four test currents. The simulated electrical resistance results are underestimated by up to $17.9 \%$ at the beginning of the simulations heat up cycle for the lower current case, while agreement improves up to $7.8 \%$ at $2500 \mathrm{~s}$ for the highest current case. All temperatures agree within $\pm 5 \%$ in steady state, except for $\mathrm{TC}_{3}$ which shows an error of $+19.4 \%$ at steady state in the 21.1 A test current. It has to be noted that the error is larger for the lower current cases and, in general, it decreases with time. Therefore, the model compares better with the experiments at higher temperatures. This suggests that misjudgements of any assumption have a less significant role in steady-state electro-thermal equilibrium. The dry tests also guarantee that the epoxy resin, used for the wet firing tests on the ceramic gasket, operates below its maximum operating temperature of $80^{\circ} \mathrm{C}$. In particular, $T C_{2}$ measures a temperature of $36^{\circ} \mathrm{C}$ after $200 \mathrm{~s}$ at the maximum test current $(I=23.6 \mathrm{~A})$, which is well within the maximum firing time. 

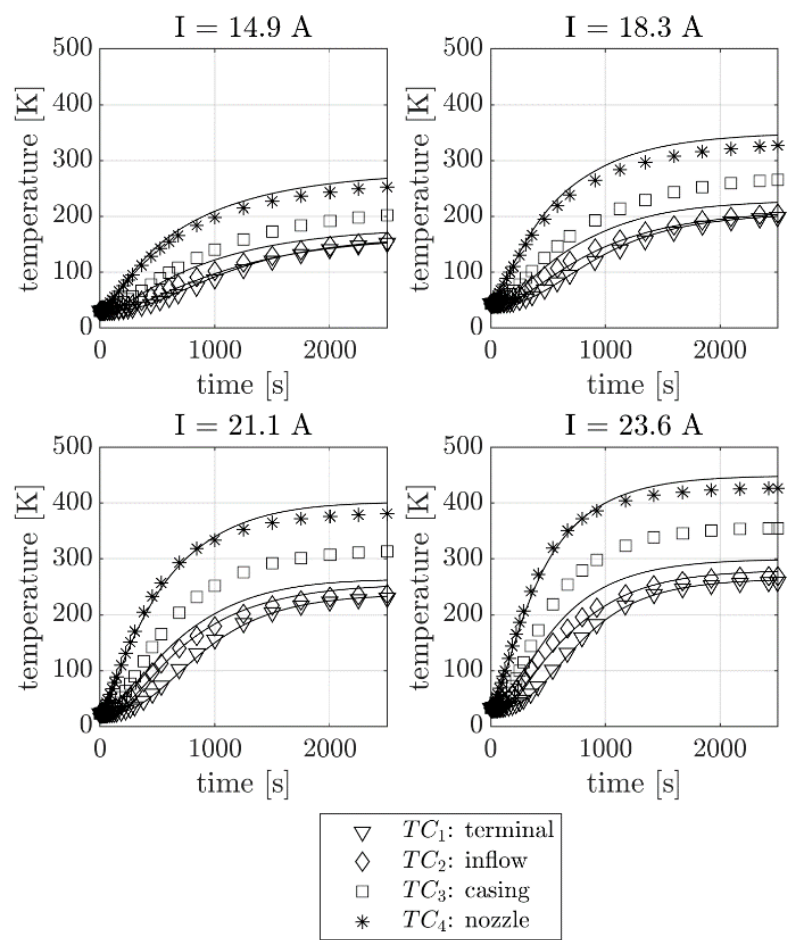

Fig. 7. Dry tests: experimental temperatures (solid lines) and 3D sector-symmetric simulations results (markers) [1 column width].

The model results are used here for further discussion. Table 5 lists the maximum temperature of the thruster, $T_{m}$, and the maximum temperature evaluated in the three regions connecting each pair of cylinders (the subscript indicates the cylinders labels as shown in Fig. 1). These regions are subjected to significant thermal stress due to the differential expansion of the cylinders caused by large thermal gradients. In addition, the maximum temperature of $316 \mathrm{~L}$ for intermittent service is of $870^{\circ} \mathrm{C}$ in air (no vacuum data available), and $925^{\circ} \mathrm{C}$ for continuous service. Thermal cycles exceeding these temperatures influence cracking and spalling. The simulations suggest that above $18 \mathrm{~A}$ of current, the connecting material between cylinders 1 and 2 could be prone to cracking during thermal cycling. The time for the heat exchanger to reach a temperature of $870^{\circ} \mathrm{C}$ is $146 \mathrm{~s}$ and $60 \mathrm{~s}$ for $I=21.1 \mathrm{~A}$ and 23.6 A respectively. This overheating process is therefore likely to be the cause of the prototype STAR-0 thermal cycling failure described in [15] at test currents of 20A and 25A.

Table 5. Dry tests model results at $t=2500 \mathrm{~s}$ : maximum structural temperature, $T_{m}$, and maximum temperature in the connectors regions.

\begin{tabular}{ccccc}
\hline$I$ [A] & $T_{\mathrm{m}}\left[{ }^{\circ} \mathrm{C}\right]$ & $T_{1-2}\left[{ }^{\circ} \mathrm{C}\right]$ & $T_{2-3}\left[{ }^{\circ} \mathrm{C}\right]$ & $T_{3-4}\left[{ }^{\circ} \mathrm{C}\right]$ \\
\hline 14.9 & 694.4 & 687.4 & 439.8 & 495.7 \\
18.3 & 861.0 & 847.7 & 549.7 & 628.1 \\
21.1 & 985.7 & 966.2 & 633.9 & 726.0 \\
23.6 & 1093.5 & 1067.5 & 707.2 & 808.5 \\
\hline
\end{tabular}

\subsection{Wet Firing Performance}

The cold firing stagnation pressure at the nozzle inlet can be estimated using an assumption of chocked flow Eq.(9), where $R$ is the specific gas constant, $\gamma$ the ratio of specific heats, $A_{t}$ the throat area and $T_{0}$ the stagnation temperature at the nozzle inlet, which is assumed to be at $300 \mathrm{~K}$. The thrust is calculated using Eq.(11), where $v_{e}, p_{e}$ and $A_{e}$ are the velocity, the static pressure and the area at the nozzle exit, while $p_{v c}$ is the measured vacuum chamber pressure. The Mach number at the exit is evaluated by calculating the zero of Eq.(10), resulting in $\mathrm{M}=11.6$.

$$
\begin{gathered}
p_{t}=\frac{\dot{m} \sqrt{T_{0}}}{A_{t}} \sqrt{\frac{R}{\gamma}\left(\frac{\gamma+1}{2}\right)^{\frac{\gamma+1}{2(\gamma-1)}}} \\
f(M)=\frac{(\gamma+1)^{-\frac{\gamma+1}{2(\gamma-1)}}}{2} \frac{\left(1+\frac{\gamma-1}{2} M^{2}\right)^{\frac{\gamma+1}{2(\gamma-1)}}}{M}-\frac{A_{e}}{A_{t}} \\
F=\dot{m} v_{e}+\left(p_{e}-p_{v c}\right) A_{e}
\end{gathered}
$$


As expected, there is a linear relationship between chamber pressure and measured thrust for the cold gas case. The comparison agrees well with the 1D isentropic assumptions described above, where the relative error between the 1D isentropic calculation and the experiments is $+7.7 \%$ at the lowest mass flow rate, decreasing to $-0.13 \%$ at the highest mass flow rate. This suggests that the nozzle behaves as expected and that the manufacturing accuracy is acceptable and in particular that the drilling of the throat to nominal diameter is sufficient to produce a good nozzle geometry [4].

Using a 100\% nozzle efficiency and 1D i.f. assumption, the calculated cold specific impulse is of 56 s. The STAR-0-B prototype performed as expected in cold gas, proving that the nozzle geometry is close to the nominal design and that the tested assembly is leak-tight. It should be noted that the efficiency of the thruster in the cold gas case is of $77 \%$ for M1-0 and $86 \%$ for M2-0. This relatively low efficiency is likely due to the fact that the thruster body is at approximately $20^{\circ} \mathrm{C}$ while the ambient temperature achieved with the cryogenic pumps is much lower. Therefore, the radiation of the thruster to the ambient was significant. This is confirmed by the simulations, which for the M1-0 case determine a radiation loss of $0.765 \mathrm{~W}$, which corresponds to the $23.7 \%$ of the total power input and compares well to the efficiency loss (column M1-0 of Table 4).

The pressure drop, $p_{d}$, between the PC and the thruster inlet is estimated for the M1 test cases. In a first nozzle 2D axisymmetric simulation, likewise in [8], the stagnation pressure at the nozzle inlet in the cold gas case is computed using a gradient-free optimisation method (Nelder-Mead), which matches the computational thrust, calculated with Eq.(5), with the experimental value. This is performed assuming that the inlet temperature is 300K. The results for M1-0 are $p_{0}=0.478$ bar and $F=9.67 \mathrm{mN}$. Secondly, for the same test case and using $p_{0}$ as the heat exchanger outlet, the full thruster $2 \mathrm{D}$ axisymmetric model determines that the pressure drop between the nozzle and the thruster inlet is only 3 mbar. Subsequently, the cold-gas full thruster axisymmetric model using the inlet thruster (Fig. 2) stagnation pressure as a control variable. Therefore, the pressure drop between the PC and the thruster inlet can be calculated as $p_{d}=p_{P C}-p_{0, \text { in }}=0.169$ mbar. The calculated pressure drop is constant for all M1 cases, in the assumption that the temperature of the pipe segment between the PC and the thruster remains constant at $300 \mathrm{~K}$.

We further analyse the test case M1-4, where the thruster showed the maximum specific impulse. The time-dependant 2D axisymmetric model of the heat exchanger uses the pressure $p_{0, t s}(t)=p_{P C}(t)-p_{d}$ as inlet $\mathrm{BC}$ and the experimental mass flow rate as the outlet BC. The nozzle is evaluated in a separate simulation using the stagnation conditions and the nozzle wall temperature profile is evaluated at the last time step of the heat exchanger simulation. Table 6 shows a summary of the results, where the simulated to experimental relative error is $+0.97 \%$ for $F,-4.88 \%$ for $\dot{m}$ and $-9.42 \%$ for $R_{t s}$. It has to be noted that in M1-4 the nozzle behaves far from the ideal case. In particular, as a result from the relatively high stagnation temperature and of the relatively low stagnation pressure on the nozzle, a dominant viscous effect determines a thick boundary layer at the nozzle diverging section with a resulting inefficiency of the gas expansion (Fig. 8, right). A Reynolds number below 6000 calculated at the throat indicates that viscous effects may be relevant [16,17]. In this test case analysed, $R e_{t}=1021$ and the efficiency of the nozzle results of only $73.6 \%$. For this reason, it is a requirement for the STAR thruster that the nozzle at the design point operates at $R e_{t} \geq 6000$, otherwise, the potential increase in performance due to the extreme stagnation temperature vanishes in an inefficient expansion of the gas. It also can be noted that the maximum simulated exit Mach number is below to the isentropic ideal case. From Eq.(7), the calculated heater efficiency is of $29.8 \%$.

Fig. 8 (left) shows the iso-thermal surface plot of M1-4 at the last time step, highlighting the maximum gas temperature, $T_{0}$, and the maximum structural temperature, $T_{m}$. The latter is located at the back of cylinder 1 and in the region of the connectors between cylinder 1 and 2 . The difference between the maximum structural and gas temperatures is of $205^{\circ} \mathrm{C}$, which is in line with the values anticipated in [8] for the STAR design using high-temperature materials. Finally, it can be noted that $T_{m}$ is below the $870^{\circ} \mathrm{C}$ intermittent usage limit for 316L, indicating that at this condition the thruster cannot operate for more than 2 minutes continuously and that a duty cycle must be applied for continuous operation, as described in [8].

Table 6. Main simulation results for M1-4 at $t_{h}=118.4 \mathrm{~s}$.

\begin{tabular}{cccccc}
\hline$p_{0}[\mathrm{bar}]$ & $T_{0}\left[{ }^{\circ} \mathrm{C}\right]$ & $T_{m}\left[{ }^{\circ} \mathrm{C}\right]$ & $\operatorname{Re}_{t}[-]$ & $M_{e}[-]$ & $\eta_{n}[\%]$ \\
\hline 0.826 & 649 & 854 & 1021 & 7.88 & 73.6 \\
\hline
\end{tabular}



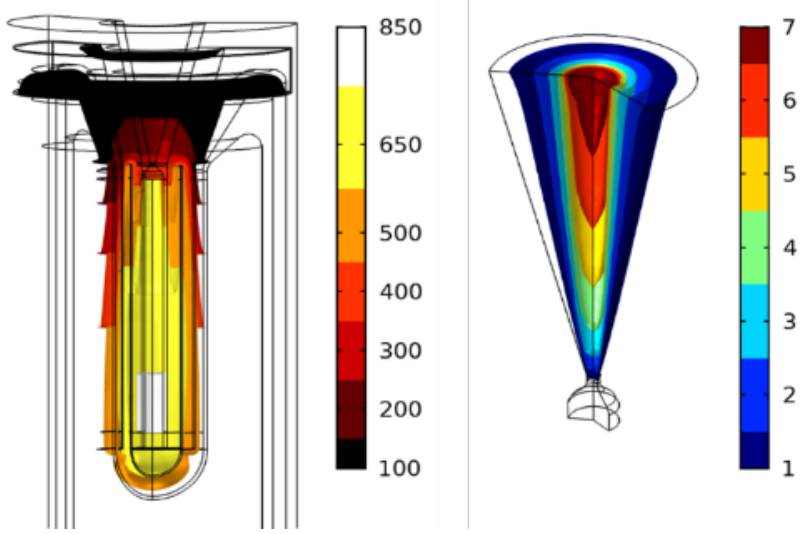

Fig. 8. M1-4 at $t_{h}=118.4 \mathrm{~s}$ : iso-thermal plot highlighting the maximum gas $\left(650^{\circ} \mathrm{C}\right)$ and structural $\left(850^{\circ} \mathrm{C}\right)$ temperatures $($ left $)$ and iso-Mach plot of the nozzle (right) [1 column width].

\section{Conclusions}

This work validates the first validation of a proof of concept prototype for the Super-high Temperature Additive Resistojet (STAR) containing an innovative multifunctional monolithic heat exchanger, enabled by selective metal laser melting 5. manufacturing processes. The STAR-0 prototype performed as expected both electrically and thermally, and produced a maximum propellant temperature comparable to the state of the art resistojets, hence validating the operational concept. A 316L stainless steel printed thruster was characterized through a combination of dry heating and wet firing tests. This includes verification testing with argon in both cold and hot firing mode, at a range of electrical power inputs. Thrust measurements ranged from $9.7 \pm 0.025 \mathrm{mN}$ to $29.8 \pm 0.025 \mathrm{mN}$, with a maximum measured specific impulse with Ar of $80.11 \pm 1.22 \mathrm{~s}$.

Multiphysics models provided insight in the temperature distribution inside the heat exchanger, in particular indicating the maximum expected structural and stagnation temperatures, which were $854^{\circ} \mathrm{C}$ and $649^{\circ} \mathrm{C}$ respectively. The degree of agreement of data with the transient simulations provides confidence of the further exploratory design simulation as discussed in [8], where STAR has been evaluated with xenon gas and candidate heat exchanger materials. The prototype model has shown a possible design flaw in the ceramic gasket, which did not guarantee a leak-tight assembly. This issue is aimed to be solved in the next design phases of the thruster. In conclusion, the first STAR prototype performed as expected and builds the basis for the next phases of the research project, such as to develop an engineering model using refractory metals and nickel alloys.

\section{Acknowledgements}

This project was funded by the Doctoral Training Partnership through the Engineering and Physical Sciences Research Council (EPSRC), grant no. EP/M50662X/1. This work was also supported by the UK Space Agency in the CEOI-ST National Space Technology Programme (NSTP-2) in partnership with Surrey Satellite Technology Limited as an end-user of the technology of the Very High Temperature Resistojet. The authors would like to thank the $\mu$-VIS lab and the EDMC workshop of the University of Southampton for the technical support and Matthew Robinson for providing valuable help with the LabVIEW interface development. All data supporting this study are openly available from the University of Southampton repository at DOI:10.5258/SOTON/D0855.

\section{References}

[1] R. Amri, D. Gibbon, In orbit performance of butane propulsion system, Adv. Sp. Res. (2012). doi:10.1016/j.asr.2011.11.021.

[2] K.T. Chojnacki, R. Reinicke, Xenon Resistojet Design and Development, in: Int. Electr. Propuls. Conf., Kitakyushu, Japan, 1999.

[3] G. Cifali, S. Gregucci, T. Andreussi, M. Andrenucci, Resistojet Thrusters for Auxiliary Propulsion of Full Electric Platforms, in: 35th Int. Electr. Propuls. Conf., 2017.

[4] F. Romei, A.N. Grubišić, D. Gibbon, Manufacturing of a High-Temperature Resistojet Heat Exchanger by Selective Laser Melting, Acta Astronaut. 138 (2017) 356-368. doi:10.1016/j.actaastro.2017.05.020.

[5] I. Coxhill, D. Gibbon, A Xenon Resistojet Propulsion System for Microsatellites, 41st AIAA/ASME/SAE/ASEE Jt. Propuls. Conf. \&amp; Exhib. 44 (2005) 1-10. doi:10.2514/6.2005-4260.

[6] A.N. Grubisic, S.B. Gabriel, Assessment of the T5 and T6 Hollow Cathodes as Reaction Control Thrusters, J. Propuls. Power. 32 (2016) 810-820. doi:10.2514/1.B35556.

[7] C. Ogunlesi, A. Grubisic, F. Romei, M. Robinson David, S. Ahmed, P. Aimone, F. Dary, D. Gibbon, M. Curtis Rouse, Novel non-destructive inspection of the STAR additively manufactured resistojet, in: Sp. Propuls. Conf., 2018. https://eprints.soton.ac.uk/417166/.

[8] F. Romei, A. Grubisic, Numerical study of a novel monolithic heat exchanger for electrothermal space propulsion, Acta Astronaut. 159 (2019) 8-16. doi:10.1016/J.ACTAASTRO.2019.03.025.

[9] T.S. Hunnewell, K.L. Walton, S. Sharma, T.K. Ghosh, R. V. Tompson, D.S. Viswanath, S.K. Loyalka, Total hemispherical emissivity of SS 316L with simulated very high temperature reactor surface conditions, Nucl. Technol. (2017). 
doi:10.1080/00295450.2017.1311120.

[10] Emissivity Table, Calex Electron. Ltd. (2019).

[11] P. Wang, Z. Xie, H. Meng, Z. Hu, Effects of the temperature and roughness on the metal emissivity, in: 27th Chinese Control Decis. Conf. (2015 CCDC), IEEE, 2015: pp. 6197-6200. doi:10.1109/CCDC.2015.7161926.

[12] K.A. Ibrahim, B. Wu, N.P. Brandon, Electrical conductivity and porosity in stainless steel 316L scaffolds for electrochemical devices fabricated using selective laser sintering, Mater. Des. 106 (2016) 51-59. doi:10.1016/J.MATDES.2016.05.096.

[13] F. Romei, A.N. Grubišić, High Performance Resistojet Thruster: STAR Status Update, in: Sp. Propuls. Conf., Seville, Spain, 2018. https://eprints.soton.ac.uk/417359/.

[14] Bronkhorst, FLUIDAT® web page, (2018).

[15] M. Robinson, A. Grubisic, F. Romei, C. Ogunlesi, S. Ahmed, P. Aimone, F. Dary, D. Gibbon, Endurance Testing of the STAR Additively Manufactured Resistojet, in: Sp. Propuls. Conf., Seville, Spain, 2018. https://eprints.soton.ac.uk/417912/.

[16] M. V Whalen, Low Reynolds Number Nozzle Flow Study, (1987).

[17] F. Romei, A. Grubisic, D. Gibbon, O. Lane, R.A. Hertford, G. Roberts, A Thermo-fluidic Model for a Low Power Xenon Resistojet, in: Jt. Conf. 30th ISTS, 34th IEPC 6th NSAT, Hyogo-Kobe, Japan, Kobe, Japan, $2015:$ p. 15. https://eprints.soton.ac.uk/401468/. 